Supporting information for ACS Sensors

\title{
Large-Area Low-Cost Plasmonic Perfect Absorber Chemical Sensor Fabricated by Laser Interference Lithography
}

\author{
Shahin Bagheri, ${ }^{\dagger}$ Nikolai Strohfeldt,${ }^{\dagger}$ Florian Sterl, ${ }^{\dagger}$ Audrey Berrier, ${ }^{\dagger}$ Andreas Tittl, ${ }^{\dagger}, \S$ \\ and Harald Giessen* ${ }^{\dagger} \dagger$ \\ ${ }^{\dagger} 4^{\text {th }}$ Physics Institute and Research Center SCoPE, University of Stuttgart, 70569 Stuttgart, Germany \\ ${ }^{\ddagger} 1^{\text {st }}$ Physics Institute and Research Center SCoPE, University of Stuttgart, 70569 Stuttgart, Germany \\ ${ }^{\S}$ Institute of BioEngineering, École Polytechnique Fédérale de Lausanne (EPFL), CH-1015 Lausanne, \\ Switzerland
}

\begin{abstract}
The Supporting Information shows: $\left(\mathrm{S}_{1}\right)$ Centroid wavelength of the large-area perfect absorber sensor for different hydrogen concentrations. (S2) Time trace of the reflection of the large-area perfect absorber sensor for different hydrogen concentrations at different wavelengths. $\left(\mathrm{S}_{3}\right)$ Time-resolved optical response of the palladium based plasmonic perfect absorber sensor with minimum reflection at $950 \mathrm{~nm}$ during exposure to hydrogen. ( $\mathrm{V}_{1}$, video file) Reflectance time trace (upper panel) as well as centroid wavelength time trace (lower panel) of large-area perfect absorber (shown in Figure 5) for different hydrogen concentrations is presented.
\end{abstract}




\section{Figure S1}

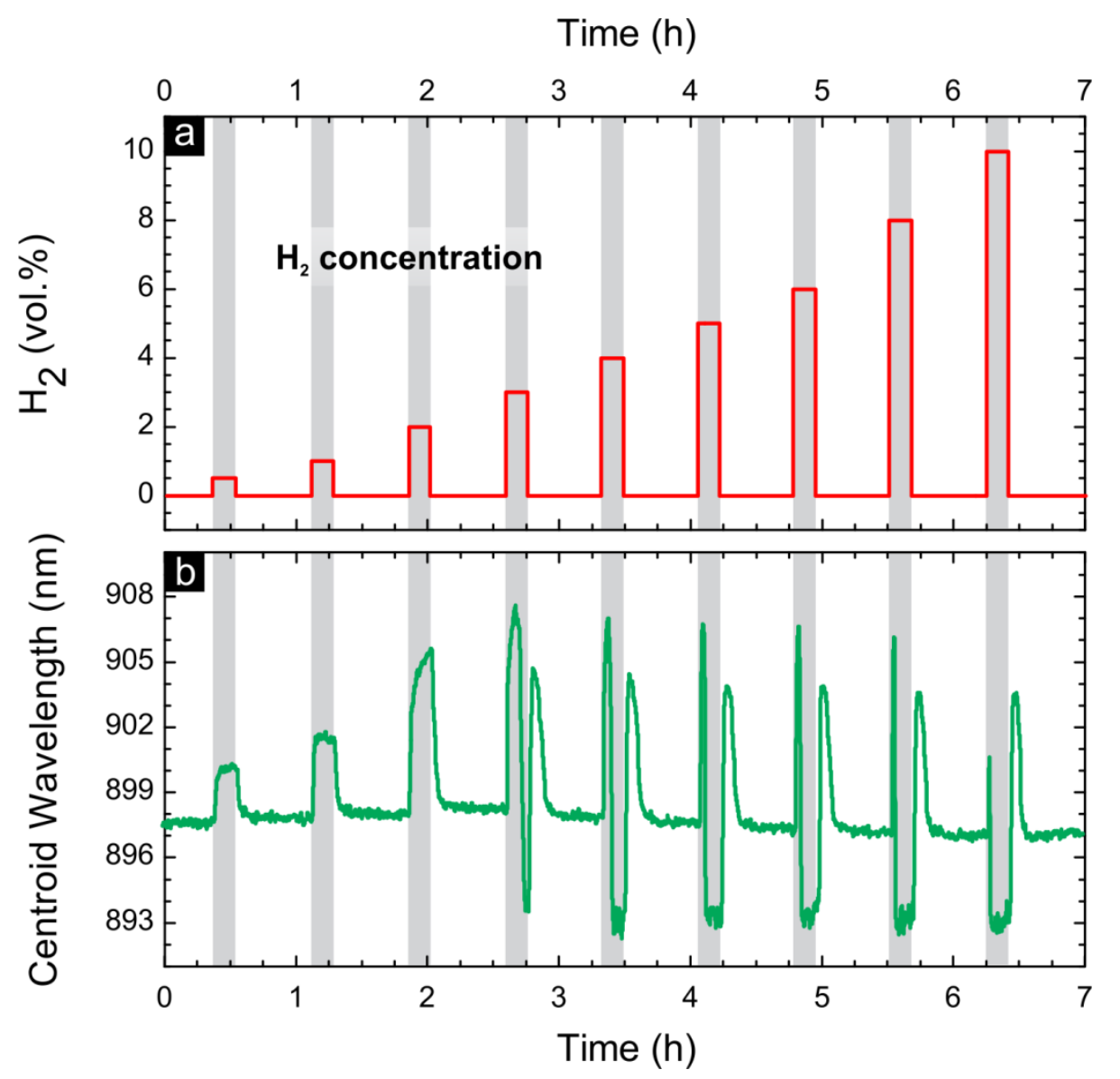

Figure S1. Optical response of the palladium based plasmonic perfect absorber sensor during exposure to different hydrogen concentrations in nitrogen carrier gas. (a) The hydrogen cycling condition is identical for all experiments. The sample is exposed to different hydrogen levels for 600 sec and to $100 \%$ N2 for 2000 sec after each hydrogen step. (b) Centroid wavelength of the large-area perfect absorber sensor for different hydrogen concentrations. The minimum reflectance initially red-shifts (Pd in $\alpha$-phase) and then shifts into the blue with strongly increasing reflectance and broadening of the spectra, when Pd transforms into the $\beta$-phase. 


\section{Figure S2}

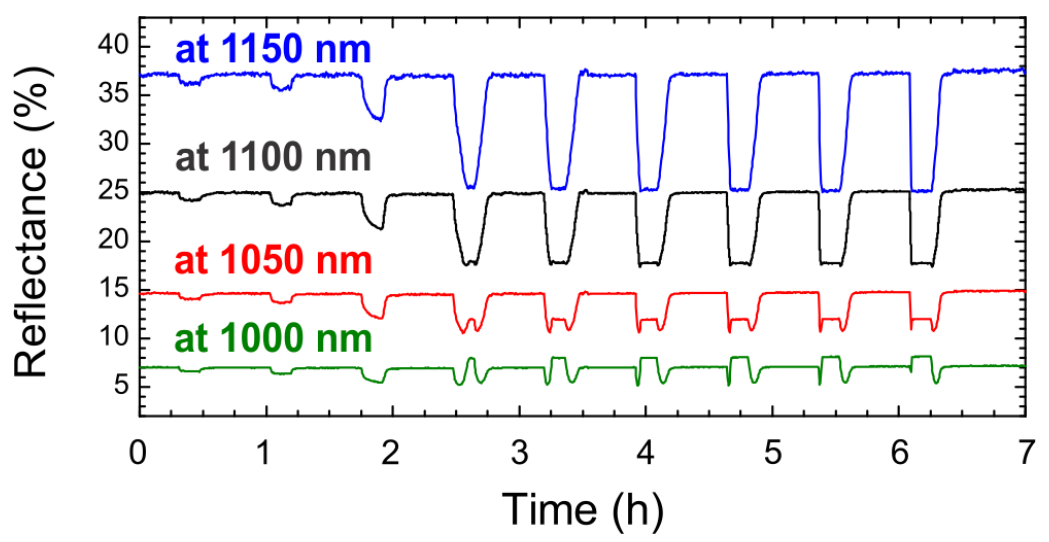

Figure S2. Time trace of the reflection of the large-area perfect absorber sensor for different hydrogen concentrations at different wavelengths. The changes in reflection become smoother when increasing the wavelength. 


\section{Figure S3}

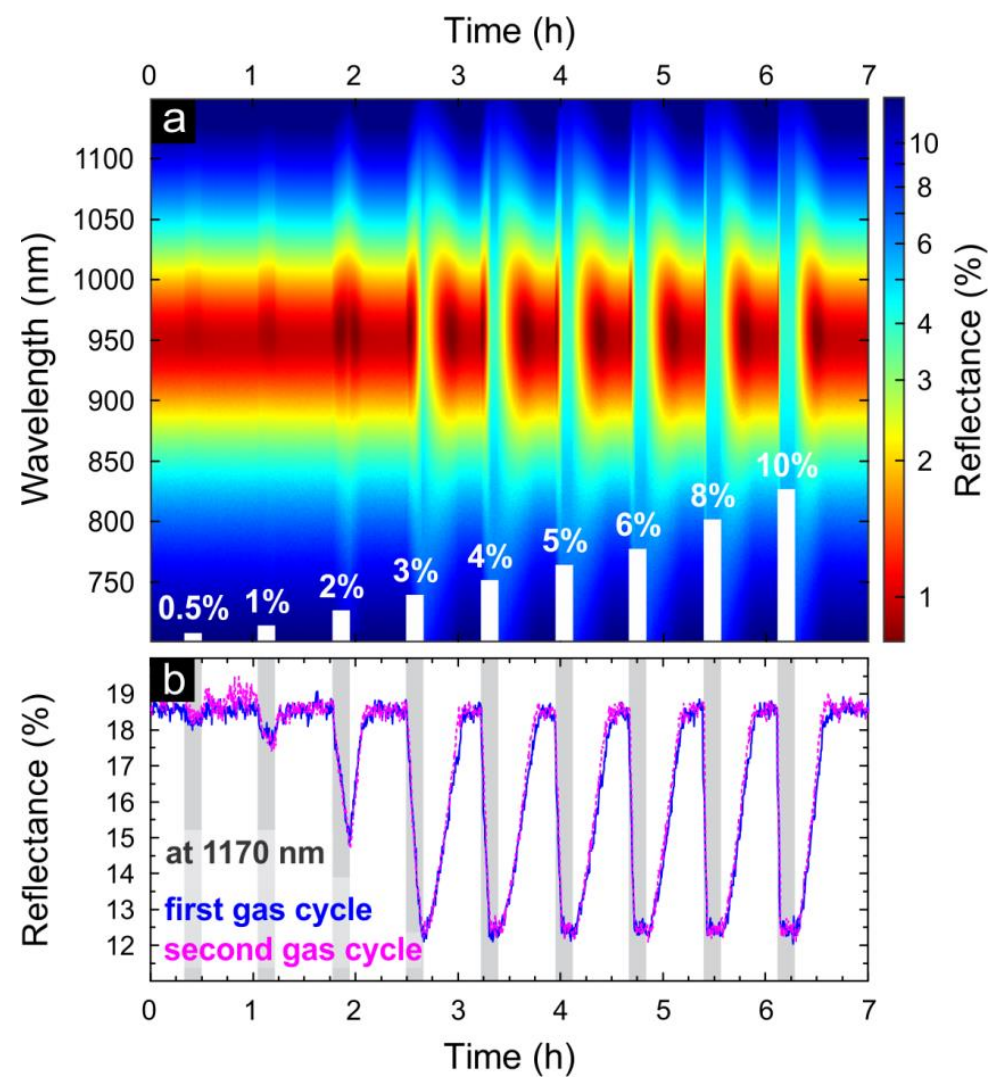

Figure S3. Time-resolved optical response of the palladium based plasmonic perfect absorber sensor with minimum reflection at $950 \mathrm{~nm}$ during exposure to hydrogen. (a) The color-coded plot of the time-dependent reflectance spectra for different hydrogen concentrations (white colored bars). The sample is exposed to different hydrogen concentrations for 600 sec and to pure nitrogen for 2000 sec after each hydrogen step. (b) Reflection of the large-area perfect absorber sensor for different hydrogen concentrations at $1170 \mathrm{~nm}$. A second measurement of the same sample is shown as dashed pink line. The small changes between the first and second sensing cycle are due to structural changes in the palladium squares. 


\section{V1 (Video File)}

Reflectance time trace (upper panel) as well as centroid wavelength time trace (lower panel) of large-area perfect absorber (shown in Figure 5) for different hydrogen concentrations is presented as a video file. The initial reflectance minimum (at $900 \mathrm{~nm}$ ) undergoes a $2 \mathrm{~nm}$ shift to higher wavelengths when applying a hydrogen concentration of 0.5 vol.\% $\mathrm{H}_{2}$ in $\mathrm{N}_{2}$ and further red-shifts when higher hydrogen concentrations are applied. The kinks in the traces (for concentrations higher than 2 vol\% $\mathrm{H}_{2}$ in $\mathrm{N}_{2}$ ) can be understood by considering the changes in palladium nanoantenna size in addition to the hydrogen-induced change of its dielectric properties (Pd in $\beta$-phase). 